\title{
Bei Standunsicherheit und häufigen Stürzen orthostatischen Tremor in Betracht ziehen
}

Fragestellung: Ziel dieser Studie war es, das klinische Bild, Charakteristika im Oberflächenelektromyogramm (EMG) und offene Behandlungsergebnisse bei einer großen Kohorte von Patienten mit orthostatischem Tremor zu erfassen.

Hintergrund: Der orthostatische Tremor ist ein sehr seltenes Krankheitsbild. Es handelt sich um einen Tremor, der als Präsentierbeschwerde eine Standunsicherheit bis hin zu Stürzen verursacht.

Patienten und Methodik: In der hier vorliegenden Untersuchung wurden retrospektiv die Befunde von 184 Patienten zusammengefasst. Die Daten wurden den entsprechenden Krankenakten entnommen.

Hassan A, Ahlskog JE, Matsumoto JY et al. Orthostatic tremor: Clinical, electrophysiologic and treatment findings in 184 patients. Neurology 2016; 86: $458-64$
Ergebnisse: In dieser Kohorte trat die Krankheit häufiger bei Frauen $(63,6 \%)$ als bei Männern auf. Die Patienten erkrankten meist um das 60. Lebensjahr herum. Die Tremorfrequenz lag zwischen 13 und $20 \mathrm{~Hz}$ (im Mittel bei 15,7). Bei 4,9\% der Patienten war die Familienanamnese positiv. Stürze ereigneten sich bei $24,1 \%$ der Patienten. Eine koexistierende Parkinson-Krankheit lag bei immerhin 8,7\% der Patienten vor, ein koexistierender essenzieller Tremor bei $22,8 \%$ der Studienteilnehmer.

Die besten Behandlungsergebnisse wurden mit Benzodiazepinen erzielt: 55,9\% von 152 Patienten berichteten über eine mäBige bis gute Besserung der Symptomatik mit Benzodiazepinen, meist Clonazepam. $31 \%$ von 42 Patienten sprachen auf die Therapie mit einem Betablocker an und $25 \%$ von 51 Patienten auf ein Antikonvulsivum, meist Gabapentin. Der Großteil der Patienten gab an, dass die Therapieeffekte mit der Zeit nachließen. Bei zwei Patienten erwies sich die tiefe Hirnstimulation (Vim) als eine effektive Behandlungsmaßnahme.

Schlussfolgerungen: Ein orthostatischer Tremor muss klinisch bei subjektiver oder objektiver Standunsicherheit vermutet werden. Als zusätzliches diagnostisches Kriterium muss im Oberflächen-EMG ein hochfrequenter Tremor $(13-20 \mathrm{~Hz})$ nachweisbar sein. Der orthostatische Tremor betrifft häufiger Frauen im fortgeschrittenen Alter und führt bei einem Viertel der Patienten zu Stürzen.

\section{- Kommentar von Günther Deuschl, Kiel}

\section{Die gängigen diagnostischen Kriterien wurden bestätigt}

Eine retrospektive Fallsammlung kann nur deshalb in einer so renommierten Zeitschrift publiziert werden, weil es sich um die größte je publizierte Serie von Patienten mit orthostatischem Tremor handelt. Sie hat die derzeit gängigen diagnostischen Kriterien bestätigt. Das EMG ist hier notwendiger Bestandteil für die Diagnose und die höhere Prävalenz bei älteren Frauen wurde bestätigt. Beachtlich ist auch der hohe Prozent-

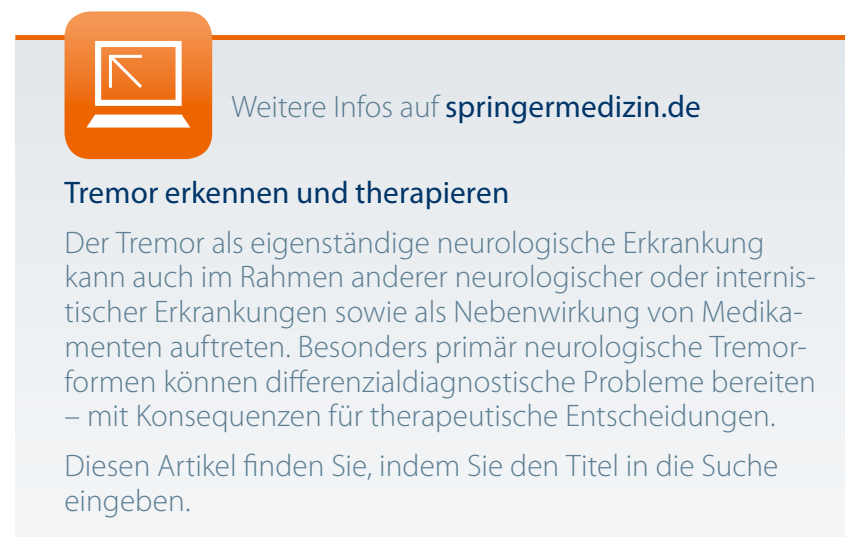

satz an Stürzen. Die teils positive Therapieantwort auf die Medikamente Gabapentin oder Primidon, Propranolol und Clonazepam wurde auch in dieser Serie bestätigt, wobei Clonazepam in der Reihenfolge wegen der Langzeitnebenwirkungen das Letzterprobte bleiben sollte. Das große Spektrum von anderen standassoziierten Tremorformen, die aber noch seltener sind, wird in dieser Arbeit leider nicht erfasst, weil sie von vornherein ausgeschlossen wurden. Differenzialdiagnostisch besonders wichtig ist der orthostatische Myoklonus, der eine sehr ähnliche klinische Präsentation hat, aber mit dem Oberflächen-EMG im Stehen ausgeschlossen werden kann.

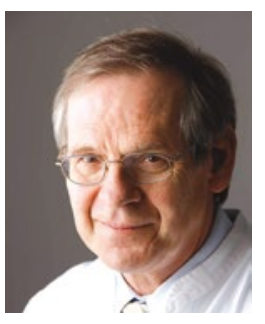

Prof. Dr. Dr. h.c. Günther Deuschl, Kiel

Emeritierter Direktor der Klinik für Neurologie, Universitätsklinikum Schleswig-Holstein, Campus Kiel (UKSH)

E-Mail: g.deuschl@neurologie.uni-kiel.de 